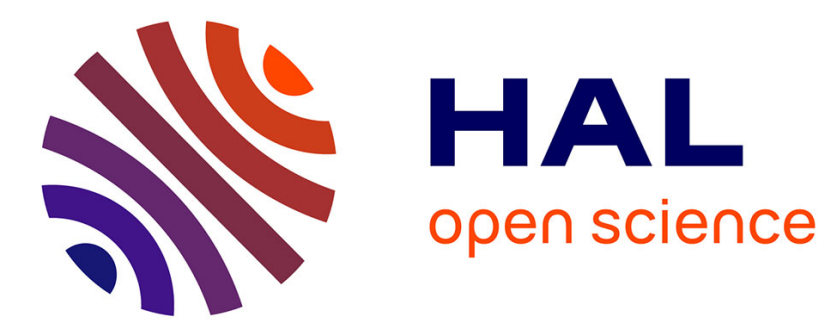

\title{
Eigenvalues and eigenvectors assignment for neutral type systems
}

Katerina V. Sklyar, Rabah Rabah, Grigory M. Sklyar

\section{To cite this version:}

Katerina V. Sklyar, Rabah Rabah, Grigory M. Sklyar. Eigenvalues and eigenvectors assignment for neutral type systems. Comptes Rendus. Mathématique, 2013, 351 (3-4), pp.91-95. 10.1016/j.crma.2013.02.007 . hal-00788702

\section{HAL Id: hal-00788702 \\ https://hal.science/hal-00788702}

Submitted on 15 Feb 2013

HAL is a multi-disciplinary open access archive for the deposit and dissemination of scientific research documents, whether they are published or not. The documents may come from teaching and research institutions in France or abroad, or from public or private research centers.
L'archive ouverte pluridisciplinaire HAL, est destinée au dépôt et à la diffusion de documents scientifiques de niveau recherche, publiés ou non, émanant des établissements d'enseignement et de recherche français ou étrangers, des laboratoires publics ou privés. 


\title{
Eigenvalues and eigenvectors assignment for neutral type systems
}

\author{
Katerina Sklyar ${ }^{\text {a }}$, Rabah Rabah ${ }^{\mathrm{b}}$, Grigory Sklyar ${ }^{\mathrm{a}}$ \\ ${ }^{a}$ Institute of Mathematics, University of Szczecin \\ Wielkopolska 15, 70-451 Szczecin, Poland \\ ${ }^{\mathrm{b}}$ IRCCyN/École des Mines de Nantes \\ 4 rue Alfred Kastler, BP 44307 Nantes, France
}

\begin{abstract}
For a class of linear neutral type systems the problem of eigenvalues and eigenvectors assignment is investigated, i.e. finding the system which has the given spectrum and almost all, in some sense, eigenvectors. The result is used for the analysis of the critical number of solvability of a vector moment problem.
\end{abstract}

\section{Résumé}

Placement de valeurs propres et de vecteurs propres pour un système avec retard de type neutre Pour une classe de systèmes linéaire avec retards de type neutre, on étudie le problème de placement de valeurs et de vecteurs propres à un nombre de vecteurs près. Le résultat est utilisé pour analyser l'intervalle critique de solvabilité d'un problème de moments vectoriel.

\section{Version française abrégée}

L'un des problème centraux de la théorie du contrôle est le placement de spectre : placement de valeurs propres mais aussi de vecteurs propres ou de structure spectrale. Nous considérons ce type de problèmes pour des systèmes linéaires avec retards de type neutre (1). Le problème de placement de vecteurs et valeurs propres se ramène de fait au problème de placement de valeurs et vecteurs singuliers pour une matrice $\Delta(\lambda)(2)$ dont les éléments sont des fonctions entières. Ces éléments spectraux sont quadratiquement proches des éléments spectraux de l'équation pour le cas $L=0$. Ces derniers étant entièrement exprimées par la structure spectrale de la matrice $A_{-1}$.

Email addresses: sklar@univ.szczecin.pl (Katerina Sklyar), Rabah.Rabah@mines-nantes.fr (Rabah Rabah), sklar@univ.szczecin.pl (Grigory Sklyar). 
Dans le présent article nous étudions le problème inverse suivant :

Quelles conditions doivent satisfaire un ensemble de nombre complexes $\{\lambda\}$ pour être les racines de l'équation caractéristique $\operatorname{det} \Delta(\lambda)=0$ et une $f a$ mille de vecteurs pour constituer le noyau de la matrice $\Delta(\lambda)$ correspondant à l'équation (1) pour un choix particulier de matrices $A_{-1}, A_{2}(\theta), A_{3}(\theta)$ ?

En fait le noyau à droite (ou le noyau à gauche) de la matrice $\Delta(\lambda)$ est lié directement aux vecteurs propres du système (1) représentés sous la forme (3) dans l'espace de Hilbert $M_{2}=\mathbb{C}^{n} \times L_{2}\left([-1,0], \mathbb{C}^{n}\right)$. Après une description détaillé des propriétés spectrales du système (3) on abouti à une caractérisation des familles de valeurs propres et vecteurs propres réalisables par un choix des matrices $A_{-1}, A_{2}(\theta), A_{3}(\theta)$.

Théorème 0.1 Soit $\mu_{1}, \ldots, \mu_{n}$ des nombres complexes distincts et $z_{1}, \ldots, z_{n}$ une famille libre de $\mathbb{C}^{n}$. Pour $\widetilde{\lambda}_{k}^{m}=\ln \left|\mu_{m}\right|+\mathrm{i}\left(\operatorname{Arg} \mu_{m}+2 \pi k\right), m=$ $1, \ldots, n, k \in \mathbb{Z}$, on considère un ensemble arbitraire de nombre complexes distincts $\left\{\lambda_{k}^{m}\right\} \cup\left\{\lambda_{j}^{0}\right\}_{j=1, \ldots, n}$ tel que $\sum_{k}\left|\lambda_{k}^{m}-\tilde{\lambda}_{k}^{m}\right|^{2}<\infty, m=1, \ldots, n$ et un ensemble arbitraire de vecteurs $\left\{w_{k}^{m}\right\} \cup\left\{w_{j}^{0}\right\}$ satisfaisant $\sum_{k}\left\|w_{k}^{m}-z_{m}\right\|^{2}<$ $\infty, m=1, \ldots, n$. Alors il existe des matrices $A_{-1}, A_{2}(\theta), A_{3}(\theta)$ telles que:

i) les nombres $\left\{\lambda_{k}^{m}, \lambda_{j}^{0}\right\}$ sont les racines simples de l'équation $\operatorname{det} \Delta(\lambda)=0$;

ii) $w_{k}^{m^{*}} \Delta\left(\lambda_{k}^{m}\right)=0, m=1, \ldots, n ; k \in \mathbb{Z}$ et $w_{j}^{0^{*}} \Delta\left(\lambda_{j}^{0}\right)=0, j=1 \ldots, n$; sauf peut être un nombre fini de vecteurs $w_{k}^{m}$ à la place desquels on obtient des vecteurs $\widehat{w}_{k}^{m}$ arbitrairement proches.

Ce résultat permet également la résolution d'un problème de moments vectoriel exprimé par les relations (11) et, plus précisément, de quantifier l'intervalle $\left(0, T_{0}\right)$ pour lequel le problème est solvable. Cet intervalle est lié à l'indice de contrôllabilité d'une paire $\left(A_{-1}, B\right)$ induite par le problème (cf. [3]).

\section{Introduction}

One of central problems in control theory is the spectral assignment problem. It is important to emphasize that the problem means the assignment of not only eigenvalues, but also eigenvectors or (in general) some geometric eigenstructure.

Our purpose is to investigate this kind of problems for a large class of neutral type systems given by the equation

$$
\dot{z}(t)-A_{-1} \dot{z}(t-1)=L z(t+\cdot), \quad t \geq 0,
$$

where $L f(\cdot)=\int_{-1}^{0}\left[A_{2}(\theta) \dot{f}(\theta)+A_{3}(\theta) f(\theta)\right] \mathrm{d} \theta, f(\theta) \in \mathbb{R}^{n}$ and $A_{-1}, A_{2}(\cdot)$, $A_{3}(\cdot)$ are $n \times n$ matrices. The elements of $A_{2}$ and $A_{3}$ are in $L^{2}(-1,0)$.

It is well known [2] that the spectral properties of this system are described 
by the characteristic matrix $\Delta(\lambda)$ given by

$$
\Delta(\lambda)=\lambda I-\lambda \mathrm{e}^{-\lambda} A_{-1}-\int_{-1}^{0}\left[\lambda \mathrm{e}^{\lambda \theta} A_{2}(\theta)-\mathrm{e}^{\lambda \theta} A_{3}(\theta)\right] \mathrm{d} \theta .
$$

In fact the problem of assignment of eigenvalues and eigenvectors is reduced to a problem of assignment of singular values and degenerating vectors of the entire matrix value function $\Delta(\lambda)$. It is remarkable $[4,5]$ that the roots of $\operatorname{det} \Delta(\lambda)=0$ are quadratically close to a fixed set of complex numbers which are the logarithms of eigenvalues of the matrix $A_{-1}$. Moreover, the degenerating vectors of $\Delta\left(\lambda_{k}\right)$ are also quadratically close to the eigenvectors of $A_{-1}$.

In this paper we investigate an inverse problem:

What conditions must satisfy a sequence of complex numbers $\{\lambda\}$ and a sequence of vectors in order to be a sequence of roots for the characteristic equation $\operatorname{det} \Delta(\lambda)=0$ and a sequence of degenerating vectors for the characteristic matrix $\Delta(\lambda)$ of equation (1) respectively for some choice of matrices $A_{-1}, A_{2}(\theta), A_{3}(\theta)$ ?

The result obtained for this problem allows to precise the critical interval for the solvability of a vector moment problem. Namely this critical interval is given by the controllability index of a couple $\left(A_{-1}, B\right)$ related to the moment problem (as considered in [3]).

\section{The operator representation and the spectral equation}

As it is shown in $[4,5]$ the system in question can be rewritten in the operator form

$$
\frac{\mathrm{d}}{\mathrm{d} t}\left(y, z_{t}(\cdot)\right)=\mathcal{A}\left(y, z_{t}(\cdot)\right),
$$

where $z_{t}(\cdot)=z(t+\cdot)$ and $\mathcal{A}: D(\mathcal{A}) \rightarrow M_{2}=\mathbb{C}^{n} \times L_{2}\left([-1,0], \mathbb{C}^{n}\right)$,

$$
D(\mathcal{A})=\left\{(y, \varphi(\cdot)) \mid \varphi(\cdot) \in H^{1}\left([-1,0], \mathbb{C}^{n}\right), y=\varphi(0)-A_{-1} \varphi(-1)\right\},
$$

and the operator $\mathcal{A}$ is given by formula $\mathcal{A}(y, \varphi(\cdot))=\left(L \varphi(\cdot), \frac{\mathrm{d} \varphi}{\mathrm{d} \theta}(\cdot)\right)$. This operator is denoted by $\widetilde{\mathcal{A}}$ instead of $\mathcal{A}$ if $A_{2}(\theta)=A_{3}(\theta) \equiv 0$. The operator $\widetilde{\mathcal{A}}$ is defined on the same domain $D(\mathcal{A})$. One can consider the operator $\mathcal{A}$ as a perturbation of the operator $\widetilde{\mathcal{A}}$, namely $\mathcal{A}(y, \varphi(\cdot))=\widetilde{\mathcal{A}}(y, \varphi(\cdot))+(L \varphi(\cdot), 0)$. Let $\mathcal{B}_{0}: \mathbb{C}^{n} \rightarrow M_{2}$ be given by $\mathcal{B}_{0} y=(y, 0)$, and $\mathcal{P}_{0}: D(\mathcal{A}) \rightarrow \mathbb{C}^{n}$ by $\mathcal{P}_{0}(y, \varphi(\cdot))=L \varphi(\cdot)$. Then $\mathcal{A}=\widetilde{\mathcal{A}}+\mathcal{B}_{0} \mathcal{P}_{0}$. Denote by $X_{\mathcal{A}}$ the set $D(\mathcal{A})$ endowed with the graph norm. The operator $\mathcal{P}_{0}$ browses the set of all linear bounded operators $\mathcal{L}\left(X_{\mathcal{A}}, \mathbb{C}^{n}\right)$ as $A_{2}(\cdot), A_{3}(\cdot)$ run over the set of $n \times n$ matrices with components from $L_{2}[-1,0]$. Indeed, an arbitrary linear operator $Q$ from $\mathcal{L}\left(X_{\mathcal{A}}, \mathbb{C}^{n}\right)$ 
can be presented as $Q(y, \varphi(\cdot))=Q_{1}\left(\varphi(0)-A_{-1} \varphi(-1)\right)+\int_{-1}^{0} \widehat{A}_{2}(\theta) \dot{\varphi}(\theta) \mathrm{d} \theta+$ $\int_{-1}^{0} \widehat{A}_{3}(\theta) \varphi(\theta) \mathrm{d} \theta$, where $\widehat{A}_{2}(\cdot), \widehat{A}_{3}(\cdot)$ are $(n \times n)$-matrices with component from $L_{2}[-1,0]$ and $Q_{1}$ is a $(n \times n)$ matrix. Let us observe that $\varphi(-1)=$ $\int_{-1}^{0} \theta \dot{\varphi}(\theta) \mathrm{d} \theta+\int_{-1}^{0} \varphi(\theta) \mathrm{d} \theta, \varphi(0)=\int_{-1}^{0}(\theta+1) \dot{\varphi}(\theta) \mathrm{d} \theta+\int_{-1}^{0} \varphi(\theta) \mathrm{d} \theta$ and denote $A_{2}(\theta)=\widehat{A}_{2}(\theta)+(\theta+1) Q_{1}-\theta Q_{1} A_{-1}$ and $A_{3}(\theta)=\widehat{A}_{3}(\theta)+Q_{1}-Q_{1} A_{-1}$. Then, the operator $Q$ may be written as $Q(y, \varphi(\cdot))=\int_{-1}^{0} A_{2}(\theta) \dot{\varphi}(\theta) \mathrm{d} \theta+\int_{-1}^{0} A_{3}(\theta) \varphi(\theta) \mathrm{d} \theta$. Hence $\mathcal{P}_{0}$ describes all the operators from $\mathcal{L}\left(X_{\mathcal{A}}, \mathbb{C}^{n}\right)$.

Assume that $\lambda_{0}$ is an eigenvalue of $\mathcal{A}, x_{0}$ is a corresponding eigenvector, and that $\lambda_{0}$ does not belong to the spectrum of $\widetilde{\mathcal{A}}$, then we have

$$
x_{0}+\left(\widetilde{\mathcal{A}}-\lambda_{0} I\right)^{-1} \mathcal{B}_{0} \mathcal{P}_{0} x_{0}=0 .
$$

Let us notice that $v_{0}=\mathcal{P}_{0} x_{0} \neq 0$, because $\lambda_{0} \notin \sigma(\widetilde{\mathcal{A}})$. Then applying the operator $\mathcal{P}_{0}$ to the left hand side of $(4)$ we get $v_{0}+\mathcal{P}_{0}\left(\widetilde{\mathcal{A}}-\lambda_{0} I\right)^{-1} \mathcal{B}_{0} v_{0}=0$. This equality means that $\lambda_{0}$ is a point of singularity of the matrix-valued function $F(\lambda)=I+\mathcal{P}_{0}\left(\widetilde{\mathcal{A}}-\lambda_{0} I\right)^{-1} \mathcal{B}_{0}$ and $v_{0}$ is a vector degenerating $F\left(\lambda_{0}\right)$ from the right. Hence, there exists a nonzero vector $w_{0}$ degenerating this matrix from the left. One can obtain the following

Proposition 2.1 Let $\lambda_{0}$ do not belong to $\sigma(\widetilde{\mathcal{A}})$. Then the pair $\left(\lambda_{0}, w_{0}\right), w_{0} \in$ $\mathbb{C}^{n}, w_{0} \neq 0$, satisfies the spectral equation

$$
w_{0}^{*} F\left(\lambda_{0}\right)=0 .
$$

if and only if $\lambda_{0}$ is a root of the characteristic equation $\operatorname{det} \Delta(\lambda)=0$ and $w_{0}{ }^{*}$ is a row-vector degenerating $\Delta\left(\lambda_{0}\right)$ from the left, i.e. $w_{0}{ }^{*} \Delta\left(\lambda_{0}\right)=0$.

Thus, one can consider the equation $w^{*} F(\lambda)=0$ as an equation whose roots $\left(\lambda_{0}, w_{0}\right)$ describe all eigenvalues and (left) eigenvectors of the characteristic matrix $\Delta(\lambda)$. We assume that the matrix $A_{-1}$ has simple nonzero eigenvalues $\mu_{1}, \ldots, \mu_{n}$. In this case $[4,5]$ the spectrum $\sigma(\widetilde{\mathcal{A}})$ consists of simple eigenvalues which we denote by $\widetilde{\lambda}_{k}^{m}=\ln _{\tilde{\lambda}}\left|\mu_{m}\right|+\mathrm{i}\left(\operatorname{Arg} \mu_{m}+2 \pi k\right), \quad m=1, \ldots, n, k \in \mathbb{Z}$, and of the zero-eigenvalue $\widetilde{\lambda}_{0}=0$. Let $\Phi=\left\{\widetilde{\varphi}_{k}^{m}\right\} \cup\left\{\widetilde{\varphi}_{j}^{0}\right\}$ be a family of almost normed eigenvectors corresponding to the eigenvalues $\left\{\tilde{\lambda}_{k}^{m}, \tilde{\lambda}_{0}\right\}$ which forms a Riesz basis in the space $M_{2}$. Denote by $\Psi=\left\{\widetilde{\psi}_{k}^{m}\right\} \cup\left\{\widetilde{\psi}_{j}^{0}\right\}$ the bi-orthogonal basis to $\Phi$. Let $w_{0}^{*}$ and $\lambda_{0}$ be as in (5) and $z_{j}, j=1, \ldots, n$ be the eigenvectors of the matrix $A_{-1}^{*}$ and let the representation of $w_{0}^{*}$ in the basis $z_{j}$ be as follows: $w_{0}^{*}=\sum_{j} \alpha_{j} z_{j}^{*}$. Thus, the condition for a pair $\left(\lambda_{0}, w_{0}\right)$ to satisfy the spectral equation, i.e. Proposition 2.1, can be rewritten in the following form.

Proposition 2.2 Let $\lambda_{0}$ do not belong to $\sigma(\widetilde{\mathcal{A}})$. Then the pair $\left(\lambda_{0}, w_{0}\right), w_{0} \in$ $\mathbb{C}^{n}, w_{0} \neq 0$, satisfies equation (5) if and only if

$$
\alpha_{m}=\sum_{k \in \mathbb{Z}} \sum_{j=1}^{n} \alpha_{j} \frac{p_{k, m}^{j}}{\widetilde{\lambda}_{k}^{m}-\lambda_{0}}, \quad m=1, \ldots, n,
$$


where for any $m, j$, the sequences $\left\{p_{k, m}^{j}\right\}$ satisfy $\sum_{k}\left|p_{k, m}^{j}\right|^{2}<\infty$.

\section{Conditions for spectral assignment}

We confine ourselves to the assignability problem of simple eigenvalues for the operator $\mathcal{A}$, this is guaranteed by the assumption that the matrix $A_{-1}$ has distincts eigenvalues (see [5]). Then one can enumerate those eigenvalues as $\left\{\lambda_{k}^{m}\right\} \cup\left\{\lambda_{j}^{0}\right\}$, for $m, j=1, \ldots, n ; k \in \mathbb{Z}$, where the sequence $\left\{\lambda_{k}^{m}\right\}$ satisfies

$$
\sum_{k, m}\left|\lambda_{k}^{m}-\tilde{\lambda}_{k}^{m}\right|^{2}<\infty
$$

Let the vectors $w_{k}^{m}$ be such that $\left(w_{k}^{m}\right)^{*} \Delta\left(\lambda_{k}^{m}\right)=0, m=1, \ldots, n ; k \in \mathbb{Z}$. Then one can also show that

$$
\sum_{k}\left\|w_{k}^{m}-z_{m}\right\|^{2}<\infty, \quad m=1, \ldots, n
$$

For all indices $m_{0}=1, \ldots, n ; k_{0} \in \mathbb{Z}$, consider decompositions $\left(w_{k_{0}}^{m_{0}}\right)^{*}=$ $\sum_{j=1}^{n} \alpha_{j m_{0}}^{k_{0}} z_{j}^{*}$. Then condition (8) for $w_{k_{0}}^{m_{0}}$ is equivalent to

$$
\sum_{k_{0}}\left|\alpha_{m m_{0}}^{k_{0}}\right|^{2}<\infty, \quad m \neq m_{0}, \sum_{k_{0}}\left|\alpha_{m m}^{k_{0}}-1\right|^{2}<\infty, \quad m, m_{0}=1, \ldots, n .
$$

We now consider the space $\ell_{2}$ of infinite sequences (columns) indexed as $\left\{a_{k}\right\}_{k \in \mathbb{Z}}$ with a scalar product defined by $\left\langle\left\{a_{k}\right\},\left\{b_{k}\right\}\right\rangle=\sum_{k} a_{k} \overline{b_{k}}$. One can also see that $\left\{\frac{1}{\tilde{\lambda}_{k}^{m}-\lambda_{k_{0}}^{m_{0}}}, k \in \mathbb{Z}\right\} \in \ell_{2}$ for all $m, m_{0}=1, \ldots, n ; k_{0} \in \mathbb{Z}$. Then, putting $\lambda_{0}=\lambda_{k_{0}}^{m_{0}}$ and $w_{0}=w_{k_{0}}^{m_{0}}$ in the equations (6), we obtain

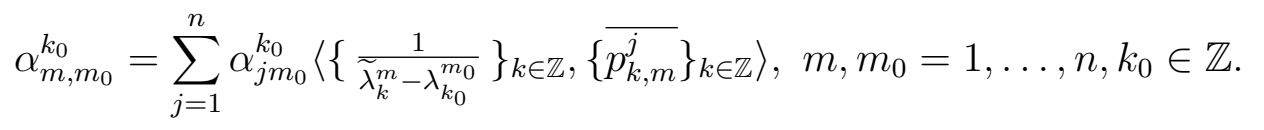

Hence, the assignment problem is equivalent to the existence of an infinite vector $\left\{p_{k, m}^{j}\right\}_{k \in \mathbb{Z}} \in \ell_{2}$ satisfying the system of equation (10).

Consider the following infinite matrices

$$
S_{m m_{0}}=\left\{\frac{1}{\widetilde{\lambda}_{k}^{m}-\lambda_{k_{0}}^{m_{0}}}\right\}_{k_{0}, k \in \mathbb{Z}}, \Lambda_{m}=\operatorname{diag}\left\{\widetilde{\lambda}_{k}^{m}-\lambda_{k}^{m}\right\}_{k \in \mathbb{Z}}, \quad m, m_{0}=1, \ldots, n .
$$

The solvability of equations (10) is based on the following result.

Lemma 3.1 1. For $m \neq m_{o}$ the infinite matrices $S_{m m_{0}}$ represent linear bounded operators from $\mathcal{L}\left(\ell_{2}\right)$ with bounded inverses.

2. $\Lambda_{m} S_{m m}$ is a bounded operator from $\mathcal{L}\left(\ell_{2}\right)$ and has a bounded inverse. 
The proof of Lemma 3.1 uses the Levin's Theorem on the property for a family of exponentials to be a Riesz basis in $L^{2}$ (see for example [1]).

Now we are ready to present our main results on the spectral assignment.

Theorem 3.2 Let $\mu_{1}, \ldots, \mu_{n}$ be different nonzero complex numbers and $z_{1}, \ldots, z_{n}$ be $n$-dimensional linear independent vectors. Denote

$$
\tilde{\lambda}_{k}^{m}=\ln \left|\mu_{m}\right|+\mathrm{i}\left(\operatorname{Arg} \mu_{m}+2 \pi k\right), \quad m=1, \ldots, n, k \in \mathbb{Z} .
$$

Let us consider an arbitrary sequence of different complex numbers $\left\{\lambda_{k}^{m}\right\}_{\substack{k \in \mathbb{Z} \\ 1 \leq m \leq n}}^{\substack{k \\ 1 \leq m}}$ such that

$$
\sum_{k \in \mathbb{Z}}\left|\lambda_{k}^{m}-\widetilde{\lambda}_{k}^{m}\right|^{2}<\infty, \quad m=1, \ldots, n,
$$

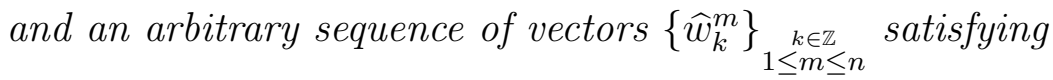

$$
\sum_{k \in \mathbb{Z}}\left\|\widehat{w}_{k}^{m}-z_{m}\right\|^{2}<\infty, \quad m=1, \ldots, n .
$$

Let, in addition, the complex numbers $\lambda_{j}^{0}, j=1, \ldots, n$ be different from each other and different from $\lambda_{k}^{m}$ and let $d_{j}^{0}, j=1, \ldots, n$ be nonzero vectors. Then, for any $\varepsilon>0$ there exist $N>0$, a sequence $\left\{w_{k}^{m}\right\}_{k \in \mathbb{Z}, m=1, \ldots, n}$ :

$$
\sum_{k \in \mathbb{Z}}\left\|w_{k}^{m}-\widehat{w}_{k}^{m}\right\|^{2}<\varepsilon, w_{k}^{m}=\widehat{w}_{k}^{m},|k|>N, m=1, \ldots, n,
$$

and a choice of matrices $A_{-1}, A_{2}(\theta), A_{3}(\theta)$ such that:

i) all the numbers $\left\{\lambda_{k}^{m}\right\}_{k \in \mathbb{Z}, m=1, \ldots, n} \cup\left\{\lambda_{j}^{0}\right\}_{j=1 \ldots, n}$ are the simple roots of the characteristic equation $\operatorname{det} \Delta(\lambda)=0$;

ii) $w_{k}^{m^{*}} \Delta\left(\lambda_{k}^{m}\right)=0, m=1, \ldots, n, k \in \mathbb{Z}$ and $w_{j}^{0^{*}} \Delta\left(\lambda_{j}^{0}\right)=0$.

As a possible application of this result, we can precise a condition for the solvability of a vector moment problem, namely by giving the time (or interval) of solvability.

Remark 3.3 Consider the following moment problem: find the function $u_{i}(t), i=1, \ldots, r$ such that

$$
s_{k}^{m}=\int_{0}^{T} e^{\lambda_{k}^{m} t}\left(b_{k, m}^{1} u_{1}(t)+\ldots+b_{k, m}^{r} u_{r}(t)\right) d t, \quad k \in \mathbb{Z}, m=1, \ldots, n,
$$

for a given sequence of complex numbers $s_{k}^{m}$. We assume that the sequence $\lambda_{k}^{m}$ verifies the conditions of Theorem 3.2 and $b_{k, m}^{j}, j=1, \ldots, r$ are such that

$$
\sum_{j, k, m}\left|b_{k, m}^{j}-b_{m}^{j}\right|^{2}<\infty, \sum_{j}\left|b_{m}^{j}\right|>0, \sum_{j}\left|b_{k, m}^{j}\right|>0, k \in \mathbb{Z}, m=1, \ldots, n .
$$

There exists $T_{0}>0$ such that this moment problem is solvable for any sequence $\left\{s_{k}^{m}\right\} \in \ell_{2}$ if $T>T_{0}$ and not solvable for $T<T_{0}$. 
Such a number is called the critical number of solvability (cf. for example [1]). In [3] it was shown that the critical number of solvability $T_{0}$ equals $n_{1}\left(A_{-1}, B\right)$, i.e. the controllability index of the couple $\left(A_{-1}, B\right)$, which is the minimal integer such that $\operatorname{rank}\left(B A_{-1} B \ldots A_{-1}^{n_{1}-1} B\right)=n$, where $B=\left\{b_{m}^{j}\right\}_{m=1, \ldots, n}^{j=1, \ldots, r}$ and $A_{-1}=\operatorname{diag}\left(\mu_{1}, \ldots, \mu_{n}\right)$, under the assumption that this problem of moments corresponds to a controllability problem of a controlled system of neutral type $\dot{z}(t)-A_{-1} \dot{z}(t-1)=L z(t+\cdot)+B u(t)$. Theorem 3.2 allows to eliminate the last assumption.

\section{Acknowledgements}

This work was partially supported by Polish National Science Center grant N N514 238438.

\section{References}

[1] Sergei A. Avdonin and Sergei A. Ivanov, Families of exponentials, Cambridge University Press, Cambridge, 1995.

[2] Jack K. Hale and Sjoerd M. Verduyn Lunel. Introduction to functionaldifferential equations, volume 99 of Applied Mathematical Sciences. SpringerVerlag, New York, 1993.

[3] Rabah R., Sklyar G. M. The Analysis of Exact Controllability of Neutral-Type Systems by the Moment Problem Approach, SIAM J. Control Optim. Vol. 46, Issue 6 (2007), 2148-2181.

[4] R. Rabah, G. M. Sklyar, and A. V. Rezounenko, Generalized Riesz basis property in the analysis of neutral type systems, C. R. Math. Acad. Sci. Paris 337 (2003), no. 1, 19-24.

[5] R. Rabah, G. M. Sklyar, and A. V. Rezounenko, Stability analysis of neutral type systems in Hilbert space, J. Differential Equations 214 (2005), no. 2, 391-428.

[6] R. Rabah, G. M. Sklyar, and P. Yu. Barkhayev, Stability and stabilizability of mixed retarded-neutral type systems, ESAIM: Control, Optimisation and Calculus of Variations, 18 (2012), 656-692. 